\title{
Uso de anti-inflamatórios não esteroides por idosos atendidos em uma Unidade de Estratégia de Saúde da Família do município de ljuí (RS)
}

\author{
Vanessa Adelina Casali Bandeira*, Camila Tais Dal Pai", Karla Renata de Oliveira"**
}

\section{Resumo}

Este é um estudo transversal e descritivo que teve como objetivo identificar os medicamentos prescritos e não prescritos aos idosos atendidos em uma Unidade de Estratégia de Saúde da Família (UESF) de ljuí (RS) e, entre eles, os anti-inflamatórios não esteroides (AINEs) utilizados para o controle da dor, bem como, o uso de AlNEs inapropriados para idosos. Os idosos foram identificados a partir dos cadastros da UESF e procurados em seus domicílios para responder um questionário constituído de perguntas abertas e fechadas relativas a características dos entrevistados, tais como, medicamentos utilizados, uso de medicamentos para a dor e se os medicamentos usados foram prescritos. Dos 116 idosos incluídos no estudo, 54,3\% eram mulheres, com média de idade de 69,1 \pm 7,7 anos. Foram identificados 409 medi- camentos em uso, desses, 25,4\% eram AlNEs, com prevalência de ibuprofeno. Verificou-se, que $79,3 \%$ dos idosos faziam uso de medicamentos para dor, e que $68,3 \%$ dos AINEs eram consumidos por automedicação, observando-se que a presença de AINEs é potencialmente inapropriados para idosos. Ressalta-se a necessidade de medidas de educação e orientação a respeito dos riscos da automedicação, principalmente quando associada à polifarmácia e ao uso de medicamentos inapropriados aos idosos. A inserção do farmacêutico nas unidades de saúde visa, portanto, orientar o uso de medicamentos, interações medicamentosas e efeitos adversos, bem como, promover o seu uso racional.

Palavras-chave: Idosos. Uso de medicamentos. Anti-inflamatórios Não esteroides. Automedicação.

* Acadêmica do curso de graduação em Farmácia, Universidade Regional do Noroeste do Estado do Rio Grande do Sul (Unijuí). Rua do Comércio, 3000 - Bairro Universitário, 98700-000, Ijuí, Rio Grande do Sul, Brasil. E-mail: vanessa.acbandeira@yahoo.com.br.

** Acadêmica do curso de graduação em Farmácia, Unijuí. Rua do Comércio, 3000 - Bairro Universitário, 98700-000, Ijuí, Rio Grande do Sul, Brasil. E-mail: camiladalpai@hotmail.com.

*** Farmacêutica Mestre, docente do Departamento de Ciências da Vida - (DCVida) - Unijuí. Rua do Comércio, 3000 - Bairro Universitário, 98700-000, Ijuí, Rio Grande do Sul, Brasil. E-mail: karla@unijui.edu.br.

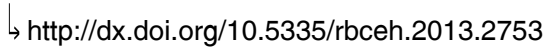




\section{Introdução}

Os anti-inflamatórios não esteroides (AINEs), encontram-se entre os agentes terapêuticos mais utilizados mundialmente, frequentemente prescritos para aliviar queixas musculoesqueléticas e utilizados, sem prescrição, no tratamento de dores menores. No entanto, praticamente todos os AINEs apresentam efeitos indesejáveis, particularmente no indivíduo idoso (RANG et al., 2003).

Os AINEs são comumente utilizados por apresentarem propriedade analgésica, antitérmica, anti-inflamatória e antitrombótica, atuam através da inibição de prostaglandinas, que são substâncias endógenas intermediárias do processo inflamatório, pela inativação das isoenzimas cicloxigenases constitutiva (COX-1) e indutiva (COX-2) (WANNMACHER; BREDEMEIER, 2004). A COX-1 está envolvida na homeostasia dos tecidos, enquanto a COX-2, é induzida nas células inflamatórias, produzindo os mediadores prostanoides da inflamação (RANG et al., 2003).

Os idosos, são grandes consumidores de AINEs, e as modificações fisiológicas relacionadas à idade podem alterar a absorção desses devido ao aumento do $\mathrm{pH}$ gástrico, o que também diminui o volume de distribuição e a capacidade de metabolização dos fármacos (ISSY; SAKATA, 2008). Destaca-se que, o declínio da função renal, pode prejudicar a excreção de outros medicamentos utilizados pelo idoso (BRASIL, 2006). Em um estudo de revisão, Batlouni (2010) apontou o aumento de riscos cardiovasculares, em especial, aqueles relacionados aos AINEs inibidores da COX-2 e, conforme o autor salienta, esse risco é maior em usuários com predisposição a doenças cardiovasculares. De acordo com Bisson (2007), esses medicamentos precisam ser indicados e administrados com cautela em idosos, pois ao prescrever um AINE para idosos, deve-se priorizar os fármacos de meia-vida curta, como o ibuprofeno, e os não acetilados, por apresentarem menos efeitos colaterais.

Mesmo diante dessas evidências, os AINEs são extensamente consumidos por idosos. Flores e Benvegnú (2008) avaliaram a utilização de medicamentos por idosos da zona urbana de Santa Rosa (RS) e verificaram que $47(5,7 \%)$ dos medicamentos eram AINEs. Ribeiro et al. (2008), ao realizarem inquérito domiciliar com idosos de Belo Horizonte (MG), observaram que os AINEs compreendiam 3,8\% dos medicamentos. É importante salientar, que o consumo de AINEs ocorre tanto através de prescrição, quanto por automedicação. Oliveira et al. (2009) verificaram, entre idosos atendidos em uma Unidade de Estratégia de Saúde da Família (UESF) de Marília (SP), que entre os medicamentos prescritos, $25(8,7 \%)$ eram AINEs. Enquanto Bortolon et al. (2008), ao analisarem a automedicação de idosas atendidas no ambulatório de Atenção ao Idoso do Hospital da Universidade Católica de Brasília (DF), verificaram que a classe mais utilizada foram os AINEs, representando $38(44,7 \%)$ dos medicamentos.

Ainda, destaca-se que, o uso de alguns medicamentos em idosos são considerados inapropriados para essa população, segundo Fick et al. (2003), por existir outra categoria com melhor 
eficácia terapêutica ou pelos seus efeitos adversos serem superiores aos benefícios (FLORES; COLET, 2010), dentre os quais encontram-se indometacina, fenilbutazona, naproxeno, piroxicam. Além disso, o uso prolongado de qualquer AINE, é considerado inapropriado para essa faixa etária (FICK et al., 2003).

O desconhecimento dos riscos quanto ao uso de medicamentos de venda livre, que incluem os AINEs e suas indicações terapêuticas levam os fármacos desse grupo a serem amplamente utilizados (LAPORTA et al., 2005), expondo os usuários a muitos riscos, principalmente os indivíduos idosos, polimedicados, estando mais suscetíveis a efeitos adversos e a interações medicamentosas.

Diante disso, o objetivo deste estudo foi identificar os medicamentos prescritos e não prescritos aos idosos atendidos em uma UESF do município de Ijuí e, entre eles, os AINEs, utilizados para o controle da dor, bem como, o uso de AINEs inapropriados para idosos.

\section{Materiais e métodos}

Foi realizado um estudo transversal, quantitativo e descritivo em uma UESF do município de Ijuí (RS).

A população do estudo foi constituída por todos os usuários, de ambos os sexos, com idade igual ou superior a sessenta anos, cadastrados em uma UESF do município. Considerando que o município no qual o estudo foi realizado é divido em microáreas de saúde, cada micro área uma organiza o cadastro da população adstrita. Desse modo, foram excluídos do estudo, aqueles com com- prometimento cognitivo ou de fala que não pudessem responder as perguntas ou que não foram encontrados em seu domicílio durante o período de coleta de dados. Aqueles que concordaram em participar do estudo, mediante assinatura o Termo de Consentimento Livre e Esclarecido (TCLE), responderam o instrumento de coleta de dados, elaborado pelas pesquisadoras, constituído por questões abertas e fechadas relativas às características dos entrevistados (idade, sexo, escolaridade, renda familiar), medicamentos utilizados, uso de medicamentos para o controle da dor, se os medicamentos usados foram prescritos, e o motivo para o uso desses medicamentos, entre outras questões.

Para a identificação dos medicamentos utilizados, foi solicitado ao idoso que apresentasse todos os medicamentos em uso, nos últimos 15 dias ou a receita médica. Assim, as informações foram obtidas por meio da observação das caixas dos medicamentos, bulas, prescrições e informações fornecidas pelo Agente Comunitário de Saúde (ACS). Os medicamentos presentes nas prescrições apresentadas pelos idosos, no momento da entrevista, foram classificados como prescritos, enquanto os medicamentos apresentados pelos idosos e não incluídos nas prescrições, foram classificados como não prescritos. Os medicamentos apresentados foram classificados no primeiro nível do sistema de Anatomical Therapeutic Chemical (ATC), enquanto os AINEs foram identificados de acordo com Wannmacher e Ferreira (2004), no caso de associações de fármacos, foi 
considerado o fármaco presente em maior quantidade. Para a classificação de medicamentos inapropriados, foram seguidos os critérios de Fick et al., 2003.

$\mathrm{O}$ protocolo do estudo foi aprovado pelo Comitê de Ética em Pesquisa (CEP) da Universidade Regional do Noroeste do Estado do Rio Grande do Sul (Unijuí) sob o Parecer Consubstanciado n. 145.1/2011.
Resultados

Dos 206 idosos cadastrados na UESF, 130 atenderam aos critérios de inclusão, desses, dez não foram localizados em seus domicílios após duas tentativas, e quatro recusaram-se a participar da pesquisa. Assim, a amostra foi constituída de 116 idosos com média de idade de 69,1 $\pm 7,7$ anos, dos quais 63 (54,3\%) eram mulheres, conforme Tabela 1.

Tabela 1 - Características sociodemográficas dos idosos entrevistados de acordo com o sexo, na cidade de Ijuí, em 2011

\begin{tabular}{lrrrr}
\hline \multirow{2}{*}{ Variável } & \multicolumn{2}{c}{ Mulheres } & \multicolumn{2}{c}{ Homens } \\
\cline { 2 - 5 } & $\mathrm{n}$ & $\%$ & $\mathrm{n}$ & $\%$ \\
\hline Idade (anos) & & & & \\
$\quad 60-69$ & 37 & 31,9 & 33 & 28,5 \\
$\quad$ 70 ou mais & 26 & 22,4 & 20 & 17,2 \\
Escolaridade (anos de estudo) & & & & \\
0 & 15 & 12,9 & 5 & 4,3 \\
1 a 4 & 38 & 32,8 & 36 & 31,0 \\
5 a 8 & 5 & 4,3 & 8 & 6,9 \\
$\quad 9$ ou + & 5 & 4,3 & 4 & 3,5 \\
\hline Fonte: dados da pesquisa. & & & &
\end{tabular}

Fonte: dados da pesquisa.

O total de medicamentos utilizados pelos entrevistados foi 409 , classificados de acordo com o sistema ATC nível 1 (Tabela 2). Desses medicamentos, 104 $(25,4 \%)$ eram AINEs, utilizados, principalmente por mulheres, evidenciado-se, em média, o uso de 0,9 AINEs por idoso. Entre os AINEs mais prescritos aos idosos encontram-se o ibuprofeno, prescrito a vinte $(19,2 \%)$ idosos, 11 $(10,6 \%)$ receberam o ácido acetilsalicílico (AAS) e dois (1,9\%), o nimesulida.
Para os medicamentos não prescritos, os quais representaram $71(68,3 \%)$ dos AINEs consumidos, verificou-se o uso de ibuprofeno por 46 idosos (44,2\%), AAS por $11(10,6 \%)$, diclofenaco por $11(10,6 \%)$ e nimesulida por três $(2,9 \%)$. Entre os AINEs utilizados pelos idosos em estudo verificou-se que $68(65,7 \%)$ foram citados pelas mulheres. 
Tabela 2 - Classificação dos medicamentos prescritos e não prescritos utilizados pelos idosos de acordo com a classe farmacológica, segundo ATC nível 1 e sexo, na cidade de Ijuí(RS), em 2011

\begin{tabular}{lcccc}
\hline & \multicolumn{2}{c}{ Feminino } & \multicolumn{2}{c}{ Masculino } \\
\cline { 2 - 5 } & $\mathrm{n}$ & $\%$ & $\mathrm{n}$ & $\%$ \\
\hline $\mathrm{N}$ - Sistema nervoso & 106 & 25,9 & 42 & 10,3 \\
C - Aparelho cardiovascular & 68 & 16,6 & 48 & 11,7 \\
M - Sistema músculo esquelético & 48 & 11,7 & 29 & 7,1 \\
A - Trato alimentar e metabolismo & 40 & 9,8 & 28 & 6,8 \\
Total & 262 & 64,0 & 147 & 36,0 \\
\hline
\end{tabular}

Fonte: dados da pesquisa.

Ainda com base na pesquisa, relataram utilizar medicamentos para dor, 92 idosos $(79,3 \%)$, principalmente mulheres $(63 \%-54,3 \%)$, das quais, cinquenta $(54,4 \%)$ apresentaram prescrição e 42 (45,6\%) declararam utilizar esses medicamentos por conta própria. Quando questionados a respeito de sentir alguma reação adversa ao uso de medicamentos, apenas quatro idosos $(4,35 \%)$ responderam afirmativamente.

$\mathrm{Na}$ sequência, quando questionados referente a ocorrência de efeitos desagradáveis, que pudessem estar relacionados ao uso dos AINEs, 47 mulheres (74,6\%) afirmaram nunca ter sentido; já, entre os homens, $32(60,4 \%)$ relataram o mesmo. Em relação a justificativa para a utilização do medicamento, trinta $(25,9 \%)$ mulheres e $19(42,2 \%)$ homens relataram sentir dor de cabeça.

Todos os AINEs citados pelos entrevistados são considerados inapropriados quando utilizados por idosos por período prolongado, sobretudo na presença de úlcera gástrica ou duodenal / com alteração na coagulação sanguínea, ou ainda, recebendo terapia anticoagulante. Além disso, o AAS na dosagem de 500mg, é inapropriado na presença de insuficiência cardíaca (FICK et al., 2003).

\section{Discussão}

Os AINEs são os fármacos mais utilizados pelos idosos que sentem dor e, muitos deles, os utilizam de forma contínua para aliviar sensações dolorosas recorrentes (LIBERMAN, 2005). Do mesmo modo, são utilizados para tratamentos sintomáticos inespecíficos, com uso amplamente difundido no mundo (WANNMACHER; BREDEMEIER, 2004).

A prevalência entre as mulheres, a faixa etária e a baixa escolaridade, evidenciados nesta pesquisa, corroboram com outros estudos realizados no Brasil. Flores e Mengue (2005) encontraram, entre os idosos de Porto Alegre (RS), prevalência de $66 \%$ de mulheres, na faixa etária entre sessenta e setenta anos, e $67 \%$ dessas, com baixa escolaridade. Marin et al. (2008), em inquérito domiciliar realizado em um município no interior paulista, verificaram que $61,8 \%$ dos ido- 
sos entrevistados eram do sexo feminino, com predomínio da faixa etária entre sessenta e 69 anos, visto que $68,1 \%$ eram analfabetos ou tinham primeiro grau incompleto. Ainda, Araújo, Magalhães e Chaimowicz (2010) verificaram, entre idosos do PSF de Belo Horizonte (MG), que $62,4 \%$ eram mulheres com média de idade de 71,8 anos.

Ressalta-se que, o uso de medicamentos eleva-se com o aumento da idade, principalmente devido à manifestação de várias doenças e respectivos agravos, além disso, varia conforme o sexo e as condições sociais e econômicas. Sabe-se que, as mulheres constituem a maioria entre os idosos, consomem mais medicamentos e frequentam serviços de saúde (BERTOLDI et al., 2004). Segundo os autores, isso ocorre porque elas apresentam maior preocupação com a saúde, procurando mais esses serviços do que os homens e, também, porque vários programas governamentais são direcionados ao cuidado com a sua saúde.

De acordo com a classificação ATC, entre os medicamentos mais utilizados, destacam-se os que atuam no sistema nervoso, seguido pelas classes utilizadas no tratamento de doenças crônicas, como os que atuam no aparelho cardiovascular, diferindo do observado por Flores e Mengue (2005) em Porto Alegre (RS), por Loyola Filho, Uchoa e Lima-Costa (2006) em Belo Horizonte (MG) e por Oliveira et al. (2009) em Marília (SP), os quais verificaram a prevalência dos medicamentos que atuam no aparelho cardiovascular, seguido pelos que atuam no sistema nervoso. Essa diferença pode estar relacionada ao questionário aplicado, o qual buscava identificar a prática da automedicação e o uso de remédios para o tratamento da dor, dentre os quais se encontram muitos fármacos que atuam no sistema nervoso.

Entre os medicamentos utilizados pelos idosos entrevistados no estudo, destacam-se os AINEs que representaram $25,4 \%$ do total utilizados, consumidos, principalmente, por mulheres, as quais, mais fazem uso do ibuprofeno. Mota et al. (2010), em São José do Rio Preto (SP), encontraram que 54\% dos medicamentos utilizados pelos idosos eram AINEs, enquanto nos estudos de Flores e Benvegnú (2008), em Santa Rosa (RS), de Oliveira et al. (2009) em Marília (SP), de Loyola Filho, Uchoa e Lima-Costa (2006) na região metropolitana de Belo Horizonte (MG), a porcentagem de idosos que utilizaram AINEs foi, respectivamente $5,7 \%, 6,4 \%$ e $2,8 \%$ dos medicamentos. Mota et al. (2010), entre os AINEs prescritos em São José do Rio Preto, encontraram a prevalência de nimesulida. Marin et al. (2008) na UBS de umacidade do interior paulista, em pesquisa domiciliar, verificaram a prevalência de diclofenaco entre os AINEs utilizados pelos idosos, diferindo do presente estudo. Acredita-se que a prevalência de ibuprofeno, em relação à nimesulida evidenciada nesta pesquisa, esteja relacionada ao fato dessa não estar incluída na Relação Municipal de Medicamentos Essenciais (Remume) do município em questão.

Os AINEs, são amplamente utilizados para diversas finalidades, entre as quais, destaca-se o controle da dor. Viletti e Sanches (2009) ao observarem o uso desses 
medicamentos entre os clientes de uma drogaria de Toledo (PR), verificaram que o seu uso tem como finalidade o tratamento de dores musculares, lombares, articulações, ou seja, dores relacionadas a algum tipo de esforço frequente. Nesse sentido, Lima e Anjos Neto Filho (2010), entre os usuários de AINEs de uma drogaria de Pimenta Bueno (RO), observaram que o principal motivo para a compra desses foi dor de cabeça em 30,5\% das respostas, semelhante a este estudo. Diante disso, é possível inferir que, independente da faixa etária, a queixa de dor é o principal motivo para o consumo de AINEs.

Os dados indicam, portanto, que os fármacos desse grupo são muito utilizados na faixa etária estudada, confirmando as referências nacionais, mesmo diante de todas as evidências relacionadas aos AINEs e as mudanças fisiológicas características dessa faixa etária, o que pode resultar em um efeito terapêutico diferenciado, marcado por grande sensibilidade, tanto aos efeitos terapêuticos quanto nos adversos dos medicamentos (NÓBREGA; KARNIKOWSKI, 2005).

Ressalta-se que o uso de AINEs, principalmente por períodos longos e sem orientação/acompanhamento, está relacionado à elevação do risco de várias complicações em idosos, especialmente naqueles que já apresentam algum tipo de comprometimento. Conforme Laine (2001), o uso prolongado de desses medicamentos aumenta o risco de úlceras gástricas. Melgaço et al. (2010), por meio de estudo de revisão, demonstram a potencial de nefrotoxidade dos AINES, especialmente em idosos com comprometimento renal prévio.
Na presente pesquisa, quando questionados em relação ao uso de medicamentos para dor, a grande maioria dos idosos respondeu de forma afirmativa e, desses, grande parte estava utilizando tais fármacos por conta própria no dia da entrevista. $\mathrm{O}$ uso de medicamentos sem prescrição médica e orientação adequada de um profissional de saúde, caracteriza automedicação, uma forma de autocuidado à saúde praticada através do consumo de um produto com o objetivo de tratar ou aliviar sintomas ou doenças (SOUSA; SILVA; NETO, 2008).

Os AINEs estão entre as classes de medicamentos mais utilizadas na prática da automedicação. Neste estudo, 68,3\% eram utilizados sem prescrição; já, em uma farmácia de Santa Maria, entre os 345 medicamentos anti-inflamatórios comercializados, $76,5 \%$ foram adquiridos sem prescrição médica (LAPORTA et al., 2005). Bortolon et al. (2008) verificaram, em um ambulatório de Atenção ao Idoso do Hospital da Universidade Católica de Brasília, que a classe que apresentou maior frequência de utilização por automedicação foi a dos analgésicos, antipiréticos e anti-inflamatórios.

Loyola et al. (2002) lembram que fatores econômicos, políticos e culturais têm contribuído para o crescimento da automedicação no mundo, tornando-a um grave problema de saúde pública. Nesse mesmo sentido, Bortolon et al. (2008) afirmam que essa prática é um fator de risco para o surgimento de problemas relacionados a medicamentos, tais como, interações medicamentosas, reações adversas, toxicidade e uso abusivo de medicamentos. 
Há que se destacar, no que se refere à manifestação de efeitos indesejados relacionados ao uso de AINEs, mais da metade, tanto das mulheres quanto dos homens, relataram que nunca sentiram sensação estranha ou diferente ao utilizar tal medicamento. Lima e Anjos Neto Filho (2010), em Pimenta Bueno (RO), ao acompanharem clientes de uma drogaria, evidenciaram que $92,1 \%$ nunca sentiram incômodo ao usar AINEs e sugerem que esse resultado esteja relacionado ao desconhecimento dos entrevistados em relação as reações adversas desses medicamentos. Dos 7,9\% que associaram os sintomas sofridos aos anti-inflamatórios ingeridos, $87,5 \%$ relataram dor de estômago, e 12,5\% cólica renal (LIMA; ANJOS NETO FILHO, 2010). Nesse sentido, sugere-se que os entrevistados também não reconheçam as reações adversas, resultando em sub-notificação das reações, o que pode ocorrer devido à idade avançada e à baixa escolaridade dos entrevistados, contribuindo para a desinformação em relação aos efeitos medicamentosos. Além disso, a falta de fornecimento de informações adequadas pelos profissionais de saúde agrava a situação, o que pode vir a ser uma estratégia do farmacêutico, profissional esse capacitado para orientar o uso de medicamentos desde que considere as limitações de entendimento dos usuários.

Destaca-se ainda, que o uso indiscriminado desses medicamentos representa risco à saúde dos usuários, pois pode resultar em interações medicamentosas e efeitos adversos (MOTA et al., 2010). Verificou-se, o uso de AINEs, potencial- mente inapropriados para idosos quando associados a determinadas patologias e utilizados por períodos prolongados, os quais possibilitam o agravamento de úlceras existentes ou provocar novas úlceras; e, em pacientes com distúrbios na coagulação sanguínea, podem prolongar o tempo de coagulação (FICK et al., 2003). No entanto, não foi investigado o tempo de utilização dos AINES, o que se constitui em uma limitação desteestudo, principalmente no que se refere aos medicamentos não prescritos, tendo relação direta com a manifestação de efeitos adversos. Também, não foram analisadas as potenciais interações medicamentosas entre AINEs e os outros medicamentos utilizados, o que é de grande relevância, considerando a idade dos entrevistados e o número de medicamentos utilizados.

No que se refere à amostra estudada, salienta-se que Bueno e Oliveira (2011), ao analisarem a Remume do município em estudo, observaram a presença do diclofenaco como inadequado para idosos quando usado por período prolongado; e, segundo os autores, a população de Ijuí está exposta a AINEs inapropriados, visto que os medicamentos incluídos nesta lista são frequentemente prescritos aos usuários do sistema público de saúde. Considerando que o ibuprofeno é o AINE mais utilizado pelos idosos entrevistados e que essa é a opção terapêutica identificada por Fick et al. (2003) para substituir o uso do diclofenaco por idosos, acredita-se que os prescritores evitavam a prescrição de medicamentos inapropriados a essa população. Ainda conforme Fick et al. (2003), como alternativa, devem ser utilizados medicamentos que 
não sejam derivados dos salicilatos, além de ibuprofeno. Esse último, segundo Issy e Sakata (2008), apresenta eliminação rápida, mesmo em indivíduos com comprometimento moderado ou intenso da função hepática e renal.

Nesse contexto, o desconhecimento de grande parte da população referente a indicação terapêutica e o uso correto do medicamento demonstra a necessidade de acesso seguro aos fármacos (LAPORTA et al., 2005). Para tanto, os autores apontam que a atuação efetiva dos profissionais da saúde é imprescindível para conscientizar e orientar a respeito do uso de medicamentos, principalmente com a inclusão da atenção farmacêutica no Sistema Único de Saúde (SUS). Ao verificar o elevado consumo de medicamentos, associado à automedicação, identifica-se a necessidade da inserção de um profissional farmacêutico nas equipes de UESF, pois esse deve promover o uso racional de medicamentos prescritos e não prescritos, tendo como objetivo informar ao usuário o modo de usar o medicamento, a via e os horários de administração, a duração do tratamento, as reações adversas, as interações, etc (MARIN et al., 2003). O uso racional de medicamentos pelos idosos é essencial para evitar gastos desnecessários, de modo a desonerar o SUS e garantir melhor qualidade de vida a esses indivíduos (NÓBREGA; KARNIKOWSKI, 2005).

A inclusão do farmacêutico na atenção básica deve ocorrer pela sua atuação no Núcleo de Apoio à Saúde da Família (NASF) que se constitui em uma equipe composta por diferentes áreas de conhecimento, atuando, juntamente com os profissionais das equipes de UESF, os quais organizam suas atividades considerando o território de sua responsabilidade e priorizando um atendimento compartilhado e interdisciplinar (BRASIL, 2009). Dessa forma, o idoso atendido por um profissional na UESF, identificado como alguém que requer orientação referente ao uso dos seus medicamentos, pode ser encaminhado ao NASF mediante agendamento.

O farmacêutico no NASF visa assegurar o acesso da população aos medicamentos seguros, eficazes e resolutivos, de forma integrada às equipes de saúde da família, principalmente por meio da assistência farmacêutica que compreende um conjunto de ações de promoção, proteção e recuperação da saúde, individual e coletiva, tendo o medicamento como insumo essencial, visando ao acesso e ao seu uso racional (BRASIL, 2009).

Além disso, a atenção farmacêutica é uma ferramenta que permite ao farmacêutico atuar na promoção da saúde, pois esse trabalha para o usuário e com o usuário, prevenindo e monitorando possíveis efeitos adversos decorrentes da terapia medicamentosa (ALMEIDA; LIMA, 2010).

\section{Considerações finais}

Constatou-se o consumo elevado de medicamentos de diferentes classes farmacológicas e que grande parte dos idosos utiliza AINEs para o controle de diferentes tipos de dor por automedicação. Verificou-se, ainda, que os AINEs usados pelos entrevistados são inapropriados para idosos se usados por tempo prolongado. A 
prevalência do uso de medicamentos que atuam no sistema nervoso e no sistema cardiovascular pode indicar que a população estudada é acometida por doenças crônicas, muitas delas, características do aumento da longevidade. Além disso, a prevalência de uso de AINEs, muitos utilizados por automedicação, indicam que a população busca formas rápidas de tratamento, principalmente relacionados ao alívio da dor.

Com isso, ressalta-se a necessidade de medidas de educação e orientação em relação aos riscos da automedicação, especialmente quando associada à polifarmácia e ao uso de medicamentos não apropriados para idosos, pois nessa faixa etária as funções fisiológicas apresentam significativas mudanças, tornando esses sujeitos mais suscetíveis aos efeitos terapêuticos e, também, aos efeitos adversos e interações medicamentosas. Nesse sentido, salienta-se que para serem efetivas, as orientações fornecidas devem ser elaboradas de acordo com o perfil da população que é predominantemente idosa com baixa escolaridade, 0 que indica a necessidade de ações de fácil entendimento e aplicabilidade. Sugere-se ainda, estudos de acompanhamento a esses indivíduos que demonstrem os efeitos colaterais e as interações medicamentosas, as quais podem se manifestar em longo prazo.

Diante do exposto, a inserção efetiva do farmacêutico nas UESF é fundamental, tendo em vista a promoção do uso racional de medicamentos e educação para evitar a automedicação. O acompanhamento farmacoterapêutico é importante para a promoção do uso racional de medicamentos, contribuindo no processo de informação ao usuário em questões como a automedicação, interrupções e substituição de medicamentos prescritos, assim como, permite a orientar a forma correta do uso do medicamento, o tempo ideal para melhora dos sintomas, a fim de evitar custos desnecessários à saúde, interações medicamentosas, efeitos adversos e polifarmácia no idoso.

Nonsteroid anti-inflammatory use for elderly treated in a unit of the family health strategy in the city of ljuí (RS)

\section{Abstract}

This is a transversal and descriptive study and the objective is to identify the medicine used by elderly treated in a Unit of the Family Health Strategy (UFHS) of ljuí (RS), in special the Nonsteroid Anti-inflammatory (NSAID), prescribed and non-prescribed to the interviewer. The elderly was selected to the FHS register and visited in their homes to to answer a questionnaire consisting of open and closed questions concerning the characteristics of respondents, drugs used, use of pain medications and if the medications used were prescribed. Of the 116 elderly enrolled in this study, $54.3 \%$ were women, with a mean age of $69.1 \pm 7.7$ years. We identified 409 medicines in use, and the nonsteroid anti-inflammatory drugs were used to $25.42 \%$, with a prevalence of ibuprofen. It was verify that $79.3 \%$ of elderly using drugs for pain and $68.3 \%$ using NSAID by self-medication, and was observed the presence of NSAID potentially inappropriate for the elderly. This study show the need for education measures and guidance on the risks of self-medication, especially when associated with polypharmacy and inap- 
propriate medication in use for elderly. The inserting of the pharmacist in Health Units seeking guidance on the use drugs, drug interactions and adverse effects, as well as promote the rational use of medicines.

Keywords: Elderly. Use of medicines. Nonsteroid anti-inflammatory drugs. Self medication.

\section{Referências}

ALMEIDA, S. M.; LIMA, G. Farmácia clínica e atenção farmacêutica. In: FERRACINI, F. T.; BORGES FILHO, W. M. Prática Farmacêutica no Ambiente Hospitalar. 2. ed. São Paulo: Ed. Ateneu, 2010. p. 307-322.

ARAÚJO, C. M. C.; MAGALHÃES, S. M. S.; CHAIMOWICZ, F. Uso de medicamentos inadequados e polifarmácia entre idosos do Programa Saúde da Família. Latin American Journal Pharmacy, Buenos Aires, v. 29, n. 2, p. $178-84,2010$.

BATLOUNI, M. Anti-inflamatórios não esteróides: efeitos cardiovasculares, cérebros-vasculares e renais. Revista Brasileira de Cardiologia, São Paulo, v. 94, n. 4, p. 556563, 2010.

BERTOLDI, A. D. et al. Utilização de medicamentos em adultos: prevalência e determinantes individuais. Revista Saúde Pública, São Paulo, v. 38, n. 2, p. 228-238, 2004.

BISSON, M. P. Farmácia clínica \& atenção farmacêutica. 2. ed. Barueri: Manole, 2007.

BORTOLON, P. C. et al. Análise do perfil de automedicação em mulheres idosas brasileiras. Ciência \& Saúde Coletiva, Rio de Janeiro, v. 13, n. 4, p. 1219-1226, 2008.

BRASIL. Ministério da Saúde. Secretaria de Atenção a Saúde. Departamento de Atenção Básica. Envelhecimento e Saúde da Pessoa Idosa. Brasília: Ministério da Saúde, 2006. 192 p. il. Cadernos de Atenção Básica. n. 9. Série A. Norma e Manuais Técnicos.
BRASIL. Ministério da Saúde. Secretaria de Atenção à Saúde. Departamento de Atenção Básica. Saúde na escola. Diretrizes do NASF - Núcleo de Apoio a Saúde da Família. Série B. Textos Básicos de Saúde. Cadernos de Atenção Básica, n. 27. Brasília: Ministério da Saúde, 2009. 160 p. il.

BUENO, C. S.; OLIVEIRA, K. R. de. Medicamentos potencialmente inapropriados para idosos: inclusão na relação municipal de medicamentos essenciais de Ijuí(RS). Revista Contexto \& Saúde, Ijuí, v. 10, n. 20, p. 299-308, jan./jun. 2011.

FICK, D. M. et al. Updating the beers criteria for potentially inappropriate medication use in older adults. Archives of Internal Medicine, Chicago, v. 163, n. 22, p. 2716-2725, 2003.

FLORES, V. B.; BENVEGNÚ, L. A. Perfil de utilização de medicamentos em idosos da zona urbana de Santa Rosa, Rio Grande do Sul, Brasil. Caderno de Saúde Pública, Rio de Janeiro, v. 24, n. 6, p. 1439-1446, jun. 2008.

FLORES, L. M.; COLET, C. F. Riscos da polifarmácia em clientes idosos. In: MALAGUTTI. W.; BERGO, A. M. A. Abordagem interdisciplinar do idoso. Rio de Janeiro: Rubio, 2010. p. 291-302.

FLORES, L. M.; MENGUE, S. S. Uso de medicamentos por idosos em região do Sul do Brasil. Revista Saúde Pública, São Paulo, v. 39 , n. 6 , p. $924-929,2005$.

ISSY, R. K.; SAKATA, A. M. Anti-inflamatórios. In: SAKATA, A. M.; ISSY, R. K. Fármacos para o tratamento da dor. Barueri: Manole, 2008. p. 1-44.

LAPORTA, L. V. et al. Avaliação da automedicação com anti-inflamatórios não esteróides em farmácias comerciais de Santa Maria (RS). Disciplinarum Scientia, Santa Maria, v. 6, n. 1, p. 1-11, 2005. Série Ciência da Saúde.

LAINE, L. Approaches to nonsteroidal anti-inflammatory drug use in the high-risk patient. Gastroenterology, v. 120, n. 3, p. 594-606, 2001. 
LIBERMAN, A. Diagnóstico e tratamento em cardiologia geriátrica. Barueri: Manole, 2005. $542 \mathrm{p}$.

LIMA, H. C.; ANJOS NETO FILHO, M. Anti-inflamatórios não esteróides e o uso indiscriminado: um estudo em uma drogaria no município de Pimenta Bueno (RO). UNINGÁ Review, Maringá, v. 4, n. 3, p. 1320, nov. 2010.

LOYOLA FILHO, A. I. de. et al. Prevalência e fatores associados à automedicação: resultados do projeto Bambuí. Revista Saúde Pública, São Paulo, v. 36, n. 1, p. 55-62, 2002.

LOYOLA FILHO, A. I. de; UCHOA, E.; LIMA-COSTA, M. F. Estudo epidemiológico de base populacional sobre uso de medicamentos entre idosos na Região Metropolitana de Belo Horizonte, Minas Gerais, Brasil. Caderno de Saúde Pública, Rio de Janeiro, v. 22, n. 12, p. 2657-2667, dez. 2006.

MARIN. N. et al. Assistência farmacêutica para gerentes municipais. Rio de Janeiro: OPAS/OMS, 2003. 373 p. il.

MARIN, M. J. S. et al. Caracterização do uso de medicamentos entre idosos de uma unidade do Programa Saúde da Família. Caderno de Saúde Pública, Rio de Janeiro, v. 24, n. 7, p. 1545-1555, jul. 2008.

MELGAÇO, S. S. C. et al. Nefrotoxicidade dos anti-inflamatórios não esteroidais. Medicina, Ribeirão Preto, v. 43, n. 4, p. 382-390, 2010.

MOTA, P. M. et al. Estudo sobre a utilização de anti-inflamatórios não esteroidais prescritos em receitas para idosos da região Noroeste Paulista. Revista de Ciências Farmacêuticas Básica e Aplicada, São Paulo, v. 31, n. 2, p. 157-163, 2010.

NÓBREGA, O. de T.; KARNIKOWSKI, M. G. de O. A terapia medicamentosa no idoso: cuidados na medicação. Ciência \& Saúde Coletiva, Rio de Janeiro, v. 10, n. 2, p. 309313, 2005.
OLIVEIRA, C. A. P. et al. Caracterização dos medicamentos prescritos aos idosos na Estratégia Saúde da Família. Caderno de Saúde Pública, Rio de Janeiro, v. 25, n. 5, p. 1007-1016, maio 2009.

RANG, H. P. et al. Farmacologia. 5. ed. Brasil: Elsevier, 2003. 905 p.

RIBEIRO, A. Q. et al. Inquérito sobre uso de medicamentos por idosos aposentados, Belo Horizonte (MG). Revista Saúde Pública, Rio de Janeiro, v. 42, n. 4, p. 724-732, 2008.

SOUSA, H. W. O.; SILVA, J. L.; NETO, M. S. A importância do profissional farmacêutico no combate à automedicação no Brasil. Revista Eletrônica de Farmácia, Goiânia, v. 5, n. 1, p. 67-72, 2008.

VILETTI, F.; SANCHES, A. C. C. Uso indiscriminado e/ou irracional de antiinflamatórios não esteroidais (AINES) observados em uma farmácia de dispensação. Visão Acadêmica, Curitiba, v.10, n.1, p. 69-76, jan./ jun. 2009.

WANNMACHER, L.; BREDEMEIER, M. Anti-inflamatórios não esteróides: uso indiscriminado de inibidores seletivos de cicloxigenase-2. Uso racional de medicamentos: temas selecionados, Brasília, v. 1, n. 2, p. 1-6, jan. 2004.

WANNMACHER L.; FERREIRAM. B. C. Anti-inflamatórios não esteróides. In: FUCHS, F.D.; FERREIRA, M. B. C.; WANNMACHER, L. Farmacologia clínica: fundamentos da terapêutica racional. 3. ed. Rio de Janeiro: Guanabara Koogan, 2004. p. 296-304. 